\title{
Erratum
}

\section{Molecular Elucidation of Two Novel Seed Specific Flavonoid Glycosyltransferases in Soybean}

Hafiz Mamoon Rehman ${ }^{1,2}$, Muhammad Amjad Nawaz ${ }^{1}$, Zahid Hussain Shah ${ }^{4}$, Gyuhwa Chung ${ }^{1, *}$ and Seung Hwan Yang ${ }^{1, *}$

Volume 61, page 320, there is an error in the author citation. Zahid Hussain $\mathrm{Shah}^{4}$ citation is Zahid Hussain Shah ${ }^{3,4}$ 